\title{
THE VALUATION STRUCTURE OF HOMOMORPHIC IMAGES OF PRÜFER DOMAINS
}

\author{
MONTE B. BOISEN, JR. AND PHILIP B. SHELDON
}

\begin{abstract}
Let $R$ denote a Prüfer ring which is a homomorphic image of a Prüfer domain $D$. The purpose of this paper is to investigate the relationship between the valuation structure over $D$ and the valuation structure over $R$. It is shown that there exists a one-to-one correspondence between the valuations over $R$ and the valuations over $D$ centered on primes containing the kernel of the homomorphism. This correspondence is shown to be natural in the sense that the value of an element of $R$ is either infinity or the value of its pre-images under the corresponding valuation over $D$. Moreover, the value group of a valuation over $R$ is an isolated subgroup of the value group of the corresponding valuation over $D$.
\end{abstract}

Introduction. Kaplansky and others have asked the following question: Is every ring with linearly ordered ideals the homomorphic image of a valuation domain? This question can be cast in a more general setting by asking whether every arithmetical ring is the homomorphic image of a Prüfer domain. Note that, when only local rings are considered, the second question reduces to the first question since a local ring is arithmetical if and only if its ideals are linearly ordered. An aid in answering these questions would be a complete description of the structure of the rings in the class of all homomorphic images of Prüfer domains. This class was studied in [1] and shown to be a proper subclass of the class of Prüfer rings. In view of the results due to Griffin (outlined below), each ring in this class possesses a valuation structure, where the valuations considered are those defined by Manis (al so discussed below).

In this paper we give a complete description of the valuation structure of a homomorphic image of a Prüfer domain $D$ in terms of the valuation structure of $D$. In $\oint_{1}$ we present a procedure that defines a natural mapping from the set of all valuations on the quotient field of $D$ which are nonnega-

Presented to the Society June 18, 1973; received by the editors June 25, 1973 and, in revised form, October 31, 1973.

AMS (MOS) subject classifications (1970). Primary 13A15, 13 F05.

Key words and phrases. Valuation, valuation pair, Prüfer rings. 
tive on $D$ and positive on the ideal $A$ to the set of all valuations on the total quotient ring of $D / A$ which are nonnegative on $D / A$. In $\$ 2$ we show that this mapping is one-to-one and onto. Specifically, we show that if this mapping is considered as a mapping from the valuation overrings of $D$ centered on prime ideals containing $A$ to the valuation pairs over $D / A$, then it agrees with the natural extension of the mapping given by the correspondence theorem for homomorphisms between prime ideals of $D$ containing $A$ and the prime ideals of $D / A$.

Throughout this paper the term "ring" will denote a commutative ring with unity. Manis [5] defines a valuation on a ring $S$ to be a mapping $w$ from $S$ onto a totally-ordered abelian group with positive infinity adjoined such that for all $a, b \in R$,

(i) $w(a b)=w(a)+w(b)$, and

(ii) $w(a+b) \geq \min \{w(a), w(b)\}$.

Note that in case $S$ is a field, then the above definition is the same as the traditional definition of a valuation. Given a valuation $w$ on $S$, the valuation ring of $w$ is defined to be the subring $W$ of $S$ of all elements with nonnegative value. The set of all elements of $S$ with positive value and the set of all elements of $S$ with infinite value are prime ideals of $W$, called the positive prime of $w$ and the infinite prime of $w$ respectively. If $V$ is a subring of $S$ and $P$ is a prime ideal of $V$, then $(V, P)$ is said to be a valuation pair of $S$ if and only if there exists a valuation on $S$ which has $V$ as its valuation ring and $P$ as its positive prime. Finally, given a ring $R,(V, P)$ is said to be a valuation pair over $R$ if $(V, P)$ is a valuation pair of the total quotient ring of $R$ and $R \subseteq V$.

Griffin [3] defines a Prüfer ring to be a ring in which every finitely generated regular ideal is invertible. (Here we use the terminology that a regular element is an element which is not a divisor of zero, and a regular ideal is an ideal which contains a regular element.). He shows that P nifer rings are related to valuation pairs through :an appropriate generalization of localization, namely, the large quotient ring. When $R$ is a ring with a total quotient ring $T$ and $Q$ is a prime ideal of $R$, the large quotient ring of $R$ with respect to $Q$ is defined to be $\{x \in T \mid$ there exists $s \in R \backslash Q$ such that $x s \in R\}$, denoted by $R_{[Q]}$. Also $[Q] R_{[Q]}$ denotes the prime ideal of $R_{[Q]}$ defined by $\{x \in T \mid$ there exists $s \in R \backslash Q$ such that $x s \in Q\}$. Griffin shows that $R$ is a Prüfer ring if and only if for every prime ideal $Q$ of $R,\left(R_{[Q]},[Q] R_{[Q]}\right)$, is a valuation pair. Thus the valuation pairs over a $P$ rüfer ring play much the same role as do the valuation overrings of a Prüfer domain. 
Our notation and terminology is essentially that of Larsen and McCarthy [4], which also contains a detailed treatment of the concepts of valuations, Prüfer rings, and large quotient rings. Where it is convenient and unambiguous to do so, we will denote the homomorphic image of an element or a set by placing a bar over its symbol.

1. We begin this section with a pair of results showing how under certain conditions a valuation on one ring can be used to construct a valuation on a closely related ring.

Proposition 1. Let $v$ be a valuation on the field $K$ with value group $G$ and valuation ring $V$. Let $I$ be a prime ideal of $V$. Then the mapping $v_{I}$ : $V_{\bar{I}} \rightarrow G \cup\{\infty\}$ defined by

$$
v_{I}(x)= \begin{cases}v(x) & \text { if } x \notin I, \\ \infty & \text { if } x \in I\end{cases}
$$

is a valuation on the ring $V_{I}$ with $V$ as its valuation ring and with an isolated subgroup of $G$ as its value group. We will call the valuation $v_{I}$ the truncation of $v$ with respect to $I$.

Proof. We will verify parts (i) and (ii) of the definition of a valuation by considering three cases. First, if $t_{1}, t_{2} \in V_{I} \backslash I$, then since $I$ is a prime ideal of $V_{I}$, we know $t_{1} t_{2} \in V_{I} \backslash I$ and hence

$$
v_{I}\left(t_{1} t_{2}\right)=v\left(t_{1} t_{2}\right)=v\left(t_{1}\right)+v\left(t_{2}\right)=v_{I}\left(t_{1}\right)+v_{I}\left(t_{2}\right) \text {. }
$$

Also when $t_{1}+t_{2} \notin I$, we have

$$
v_{I}\left(t_{1}+t_{2}\right)=v\left(t_{1}+t_{2}\right) \geq \min \left\{v\left(t_{1}\right), v\left(t_{2}\right)\right\}=\min \left\{v_{I}\left(t_{1}\right), v_{I}\left(t_{2}\right)\right\}
$$

When $t_{1}+t_{2} \in I$, then $v_{l}\left(t_{1}+t_{2}\right)=\infty$, so the condition $v_{I}\left(t_{1}+t_{2}\right) \geq$ $\min \left\{v_{I}\left(t_{1}\right), v_{I}\left(t_{2}\right)\right\}$ is automatically satisfied. Next we consider the case where $t_{1} \in I$ and $t_{2} \in V_{I} \backslash I$. Since $t_{1} t_{2} \in I$, we have

$$
v_{I}\left(t_{1} t_{2}\right)=\infty=v_{I}\left(t_{1}\right)+v_{I}\left(t_{2}\right) \text {. }
$$

Since $t_{1} \in I$ and $t_{2} \notin I$, we know that $v\left(t_{1}\right)>v\left(t_{2}\right)$ and so

$$
v\left(t_{1}+t_{2}\right)=v\left(t_{2}\right)=v_{I}\left(t_{2}\right)=\min \left\{v_{I}\left(t_{1}\right), v_{I}\left(t_{2}\right)\right\}
$$

But $t_{1}+t_{2} \notin I$ and hence $v_{i}\left(t_{1}+t_{2}\right)=v\left(t_{1}+t_{2}\right)$. Finally, in case $t_{1}, t_{2} \in I$, the $v_{I}$-values of $t_{1}, t_{2}, t_{1} t_{2}$, and $t_{1}+t_{2}$ are all $\infty$; hence both parts of the definition are trivially satisfied. 
All that remains is to show that $v_{I}$ maps $V_{I}$ onto an isolated subgroup of $G$. Since $V_{I} \backslash I$ is the group of units of $V_{I}, v_{I}$ maps onto a subgroup $H$ of $G$. The facts that $H$ is an isolated subgroup of $G$ and that $V$ is the valuation ring of $v_{I}$ both follow immediately from the definition of $v_{l}$.

Proposition 2. Let $v$ be a valuation on the ring $U$ and let $\theta: U \rightarrow \bar{U}$ be a homomorphism of $U$ onto the ring $\bar{U}$ such that the kernel of $\theta$ is contained in the infinite prime of $v$. Then $\bar{v}$ defined by $\bar{v}(\theta(u))=v(u)$ is a valuation on $\bar{U}$ with the same value group as that of $v$. In this case, we say that $v$ induces the valuation $\bar{v}$ through $\theta$.

Proof. We first show that $\bar{v}$ is well defined by showing that if $v\left(u_{1}\right) \neq$ $v\left(u_{2}\right)$, then $\theta\left(u_{1}\right) \neq \theta\left(u_{2}\right)$. If $v\left(u_{1}\right) \neq v\left(u_{2}\right)$, then by the same argument as for valuations on a field, one can show that $v\left(u_{1}-u_{2}\right)=\min \left\{v\left(u_{1}\right), v\left(u_{2}\right)\right\}$. Moreover, this minimum value cannot be $\infty$, so $v\left(u_{1}-u_{2}\right) \neq \infty$. Hence $u_{1}$ $u_{2} \notin \operatorname{ker} \theta$, so $\theta\left(u_{1}\right) \neq \theta\left(u_{2}\right)$. The remaining parts of the proof-namely that $\bar{v}$ satisfies parts (i) and (ii) of the definition of a valuation and that $\bar{v}$ maps $\bar{U}$ onto the value group of $v$-are routine to verify and will be omitted.

Before we state the main result of this section, we will establish the notation that will be used for the rest of the paper. Let $D$ denote a Prüfer domain with quotient field $K$, and let $\Psi: D \rightarrow R$ be a homomorphism of the ring $D$ onto the ring $R$ with kernel $A$. Let $T$ denote the total quotient ring of $R$. We define the multiplicative system $S$ in $D$ to be the set $\{d \in D \mid \Psi(d)$ is a regular element in $R$ \}. Boisen and Larsen [1] show that $\Psi$ can be extended to a homormophism $\phi$ of $D_{S}$ onto $T$ by defining $\phi(d / s)=\Psi(d) / \Psi(s)$.

As is customary in the theory of valuations on a field, we will consider two valuations $v$ and $v^{\prime}$ on a ring to be the same if they differ by an orderisomorphism of their value groups. In general, this condition is equivalent to saying that $v$ and $v^{\prime}$ have the same valuation pair, and in the case of valuations on a field, this condition reduces to the equality of the respective valuation rings. Now we define $\mathcal{O}$ to be the set of all distinct valuations on $K$ which are nonnegative on $D$ and positive on $A$. In other words, $v \in \mathcal{O}$ if and only if the valuation ring of $v$ contains $D$ and the center of $v$ on $D$ contains $A$. We define $W$ to be the set of all valuations of $T$ which are nonnegative on $R$. The following theorem shows how elements of $\mathcal{O}$ are related in a natural way to elements of 60 .

Theorem 1. If $v \in \mathcal{O}$, then there exists a truncation of $v$ which is defined on all of the elements of $D_{S}$ and which, when restricted to $D_{S}$, induces 
a valuation $w$ in 60 through $\phi$. This truncation of $v$ is uniquely determined and hence the process defines a mapping from 0 to 60 . Furthermore, the value group of $w$ is an isolated subgroup of the value group of $v$.

Proof. Let $v \in \mathcal{O}$ and let $V$ be its valuation ring. We note that the multiplicative system $S$ of $D$ is also a multiplicative system of $V$. Therefore, there exists a prime ideal $I$ of $V$ that is maximal with respect to not intersecting $S$. Take the truncation $v_{I}$ of $v$ with respect to $I$. Then by Proposition $1, v_{I}$ is a valuation on $V_{I}$ with value group $H$ an isolated subgroup of the value group of $v$. Since $I \cap S=\varnothing$ and $D \subseteq V$, we conclude that $D_{S} \subseteq V_{r}$.

Let $u$ denote the restriction of $v_{I}$ to $D_{S}$. We claim that $u$ is a valuation on $D_{S}$. To see this, note that since $u$ is the restriction of a valuation, it satisfies parts (i) and (ii) of the definition of a valuation. Hence we need only show that $u$ maps $D_{S}$ on to a group to complete the requirements of the definition. In fact, we will show that $u$ maps $D_{S}$ onto $H$. First we observe that an element of $V$ has its value in $H$ if and only if it is not an element of I. If $h$ is a nonnegative element of $H$, then there exists $t \in V$ such that $v(t)=h$. Since $V=D_{P}$ for some prime ideal $P$ of $D$, we have that $t=d / r$ where $d, r \in D$ and $v(r)=0$; hence $v(t)=v(d)=h$. Since $v(d) \in H, d \notin I$ and therefore $v_{I}(d)=u(d)=h$. Consequently, every positive el ement of $H$ is an image of an element of $D$. Now let $h$ be a negative element of $H$. Consider $J=\{x \in V \mid v(x) \geq-h\}$. Then $J$ is an ideal of $V$ that properly contains the ideal $I$. Therefore, by the choice of $I$ there exists an element $s \in S \cap J$. So $u(s) \geq-h$. Siruce $u(s)+b$ is a nonnegative element of $H$, there exists $d \in D$ such that $u(d)=u(s)+h$. Hence $d / s$ is an element of $D_{s}$ satisfying $u(d / s)=h$.

Now by Proposition 2, there exists a valuation $w$ on $T$ induced by $u$ through $\phi$. Furthermore, the value group of $w$ is $H$, which we noted earlier to be an isolated subgroup of $G$.

All that remains is to show that the truncation defined earlier in the proof is unique. In other words, we must show that if $J$ is a prime ideal of $V$ not equal to $I$, then the truncation $v_{J}$ does not satisfy the conditions stated in the first sentence of the theorem. Suppose that $I \varsubsetneqq J$. Then by the choice of $I$, there exists $s \in S \cap J$. Hence $1 / s \in D_{S} \backslash V_{J}$, and so the truncation $v_{J}$ does not satisfy the condition of being defined on all of the elements of $D_{s}$. On the other hand, suppose that $J \varsubsetneqq I$. If $i \in I \backslash J$, then $v(i)>v(s)$ for all $s \in S$ since $I$ is an ideal of $V$ and $I \cap S=\varnothing$. Choose $d \in D$ such that $v(d)=v(i)$. Then $d \notin J$ and hence $v_{J}(d) \neq \infty$ and $v_{J}(d)>v_{J}(s)$ for all 
$s \in S$. Let $g=v_{J}(d)$. If $e / s$ is an element of $D_{S}$ such that $v_{J}(e / s)=-g$, then we have

$$
v_{J}(s)=v_{J}(e)+g=v_{J}(e)+v_{J}(d) \geq v_{J}(d)>v_{J}(s),
$$

which is not possible. So $-g$ is not the image under $v_{J}$ of any element of $D_{S}$. If $w$ were a valuation on $T$ induced by the restriction of $v_{J}$ to $D_{S}$, then $w(\bar{d})=g$; but no element of $T$ maps to $-g$ under $w$. By definition, a valuation must map onto a group, so we have reached a contradiction. Again we conclude that the truncation $v_{J}$ does not satisfy the conditions in the first sentence of the theorem, and the proof of the uniqueness of $v_{I}$ is complete.

2. In this section we will continue with the notation developed in the preceding section. Our goal is to establish the relationship between the valuation rings belonging to elements of $\mathcal{O}$ and the valuation pairs belonging to elements of $\mathcal{W}$. In this direction, we will let $\mathcal{P}$ denote the set of all prime ideals of $D$ containing $A$. Since $D$ is a Prüfer domain, the set $\left\{D_{P} \mid P \in \mathcal{P}\right\}$ is the set of all valuation overrings of $D$ with centers containing $A$, and thus we have a natural one-to-one correspondence between $\mathcal{P}$ and $\mathcal{O}$. Now let 2 denote the set of all prime ideals of the Prüfer ring $R$. We wish to establish a one-to-one correspondence between 2 and $(1)$ analogous to the one between $\mathcal{P}$ and $\mathcal{O}$. For this purpose we need the result of the following lemma, which is probably well known but does not seem to appear in the literature in this form.

Lemma. Let $B$ be a Prüfer ring. Then every valuation pair $(W, N)$ over $B$ is of the form $\left(B_{[U]},[U]_{B}[U]\right.$, for some prime ideal $U$ of $B$.

Proof. Let $(W, N)$ be a valuation pair over $B$ and let $U$ denote the prime ideal $N \cap B$ of $B$. Then $\left(B_{[U]},[U]_{[U]}\right)$ is a valuation pair over $B$, since $B$ is a Prüfer ring. But valuation pairs can be characterized as maximal pairs with respect to the relation of "domination"' [4, Theorem 10.6, p. 229]. So in order to show $\left(B_{[U]},[U]_{B}[U]\right)=(W, N)$, it suffices to show that $(W, V)$ dominates $\left(B_{[U]},[U] B_{[U]}\right)$, in other words, that $B_{[U]} \subseteq W$ and $N \cap B_{[U]}=$ $\left[U B_{[U]}\right.$. First choose $t \in B_{[U]}$. Then there exists $b \in B \backslash U$ such that $b t$ is in $B$, hence in $W$. By definition of $U, b \in W \backslash N$ and hence $b$ has zero value. So the value of $t$ equals the value of $b t$ and consequently, $t \in W$.

To show that $B_{[U]} \cap N=[U]_{[}[U]$, it is sufficient to show that $[U]_{[}[U]$ is precisely the set of all elements of $B_{[U]}$ with positive value relative to 
the valuation of $(W, N)$. :Suppose that $x \in[U]_{[U]}$. Then there exists an element $s \in B \backslash U$ such that $x s \in U$. Since $s$ has zero value and $x s$ has positive value, it follows that $x$ has positive value. Now suppose that $y$ is an element of $B_{[U]}$, with positive value. Then there exists $r \in B \backslash U$ such that $r y \in B$. But $r y$ has the same positive value as $y$. Therefore $r y \in U$ and hence $y \in[U] B[U] \cdot$ Q.E.D.

From this lemma we conclude that each valuation pair over $R$ arises, in a natural way, from a prime ideal of $R$. Moreover, the fact that distinct prime ideals of $R$ yield distinct valuation pairs follows from the fact that $[Q] R_{[Q]} \cap R=Q$ for any prime ideal $Q$ of $R$. Consequently, there exists a natural one-to-one correspondence between 2 and $\mathcal{W}$ pairing the prime ideal $Q$ with the valuation $w$ that has valuation pair $\left(R_{[Q]},[Q] R_{[Q]}\right)_{i}$ : This correspondence, together with the correspondence between $\mathcal{P}$ and $\vartheta$ mentioned earlier, will be used in the proof of the following theorem, which is the main result of this section.

Theorem 2. Let $v$ be a valuation in $\mathcal{O}$, and let $w$ be the valuation in (1) which is associated with $v$ under the mapping defined in Theorem 1. If $P$ is the prime ideal in $\mathcal{P}$ such that $D_{P}$ is the valuation ring of $v$, and if $Q=\bar{P}$, then the valuation pair of $w$ is $\left(R_{[Q]},[Q] R_{[Q]}\right)$ : Consequently, the mapping defined in Theorem 1 is a one-to-one correspondence from 0 onto 60 .

Proof. Let $v \in \mathcal{O}$ and let $P$ be the prime ideal in $\mathcal{P}$ for which $D_{P}$ is the valuation ring of $v_{\text {. }}$ Let $C$ denote $D_{P} \cap D_{S}$. It is clear that $C$ is the set of all el ements in $D_{S}$ with nonnegative value under $v_{I}$. Since $w$ is induced by the restriction of $v_{I}$ to $D_{S}$ through $\phi, \bar{C}$ is precisely the set of all elements of $T$ with nonnegative value under $w$. In other words, $\bar{C}$ is the valuation ring of $w$. We will show that $\bar{C} \cdot=R_{[Q]}$, where $Q=\bar{P}$. First, let $\bar{c} \in \bar{C}$. Then $c=d / b$, where $d \in D$ and $b \in D \backslash P$. Then $\bar{b} \in R \backslash Q$ and $\bar{b} \bar{c}=$ $\bar{d}$ which is in $R$. Hence $\bar{c} \in R_{[Q]}$, and $\bar{C} \subseteq R_{[Q]}$.

To show the reverse containment, we let $t \in R_{[Q]} \cdot$ Then there exists $r \in R \backslash Q$ such that $r t \in R$. Since $\phi$ maps $D_{S}$ onto $T$, there exist $r^{\prime}$ and $t^{\prime}$ in $D_{S}$ such that $\phi\left(r^{\prime}\right)=r$ and $\phi\left(t^{\prime}\right)=t$. Now let $D^{\prime}$ and $P^{\prime}$ denote $\phi^{-1}(R)$ and $\phi^{-1}(Q)$ respectively. Then it is clear that $D^{\prime}$ is a subring of $D_{S}$ containing $D$, and $P^{\prime}$ is a prime ideal of $D^{\prime}$. Moreover, our choice of $r^{\prime}$ and $t^{\prime}$ shows that $r^{\prime} t^{\prime} \in D^{\prime}$ and $r^{\prime} \in D^{\prime} \backslash P^{\prime}$. So $t^{\prime} \in D_{P^{\prime \prime}}^{\prime}$. But since $D^{\prime}$ is an over-ring of the Prüfer domain $D$, we know that $D_{P^{\prime}}^{\prime}=D_{\left(P^{\prime} \cap D\right)}$ [2, Theorem 26.1, p. 322]. : Since $Q=\bar{P}$ and $P^{\prime}=\phi^{-1}(Q)$, it follows that $P^{\prime} \cap D=P$. So $t^{\prime} \in D_{P} \cap D_{S}=C$, and thus $t=\bar{t}^{\prime} \in \bar{C}$, which is what we needed to show. 
Next consider the positive prime $M$ of $w$. By the definition of $w$, it is clear that $M$ is the image of $P D_{P} \cap D_{S}$ under $\phi$. By obvious modifications of the computations that showed $\bar{C}=R_{[Q]}$, one can show that $M=[Q] R_{[Q]}$. We have now completed the proof that $w$ has $\left(R_{[Q]},[Q] R_{[Q]}\right)$ as its valuation pair.

To prove the final statement in the theorem, we first consider the oneto-one correspondence between $\mathcal{O}$ and $\mathcal{P}$ and that between 2 and $\mathcal{O}$ mentioned earlier. Composing these with the natural one-to-one correspondence between $\mathcal{P}$ and $\mathcal{Q}$ that arises from the correspondence theorem of homomorphisms, we obtain a one-to-one correspondence from $\mathcal{O}$ to 20 . Specifically, if $v \in \mathcal{O}$ with valuation ring $D_{P}$, then $v$ corresponds to $w \in W$ with valuation pair $\left(R_{[Q]},[Q] R_{[Q]}\right)$, where $Q=\bar{P}$. But by the first part of this theorem, it is clear that this correspondence is precisely the mapping defined in Theorem 1. Q.E.D.

\section{REFERENCES}

1. Monte B. Boisen, Jr. and Max D. Larsen, On Prüfer rings as images of Prüfer domains, Proc. Amer. Math. Soc. 40 (1973), 87-90.

2. Robert Gilmer, Multiplicative ideal theory, Dekker, New York, 1972.

3. Malcolm Griffin, Prüfer rings with zero divisors, J. Reine Angew. Math. 239/240 (1969), 55-67. MR 41 \# 188.

4. Max D. Larsen and Paul J. McCarthy, Multiplicative theory of ideals, Academic Press, New York, 1971.

5. Merle E. Manis, Extension of valuation theory, Bull. Amer. Math. Soc. 73 (1967), 735-736. MR 36 \#1436.

DEPARTMENT OF MATHEMATICS, VIRGINIA POLYTECHNIC INSTITUTE AND STATE UNIVERSITY, BLACKSBURG, VIRGINIA 24061 